Global Mental Health

cambridge.org/gmh

\section{Other \\ Letter to the Editor}

This is a cross-sectional study.

Cite this article: Kakaje A, Fadel A, Ghareeb A, Al Zohbi R (2021). Mental distress in ongoing conflict and non-conflict settings during COVID-19: a study on Syrians in different countries. Global Mental Health 8, e12, 1-3. https://doi.org/10.1017/gmh.2021.12

Received: 18 September 2020 Revised: 21 March 2021 Accepted: 30 March 2021

Author for correspondence: Ameer Kakaje,

E-mail: ameer.kakaje@hotmail.com

\title{
Mental distress in ongoing conflict and non-conflict settings during COVID-19: a study on Syrians in different countries
}

Ameer Kakaje ${ }^{1}$ D, Ammar Fadel ${ }^{1}$, Ayham Ghareeb ${ }^{1}$ and Ragheed Al Zohbi ${ }^{2}$

${ }^{1}$ Faculty of Medicine, Damascus University, Damascus, Syria and ${ }^{2}$ Faculty of Medicine, Aleppo University, Aleppo, Syria

Dear Editor,

Syria has suffered for 9 years from war which caused more than half of the children and adults to suffer from severe post-traumatic stress disorder (PTSD) and/or mental distress (Kakaje et al., 2020a,b). Even when leaving the country and being refugees, around $25 \%$ of people who left the country have suffered from post-traumatic stress symptoms (PTSS), depression, and/or anxiety (Georgiadou et al., 2018). This study evaluates the mental distress in Syrians who left the country because of war and in those who stayed in the conflict in Syria in the last years.

As self-reporting measures tend to overestimate symptoms, scores were used rather than cut-off points. Only Syrians were included, either living in Syria or those who left Syria due to war regardless of the method and Visa status and they were recruited using social media groups via online questionnaires. No participants living in camps were included. For each participant living outside Syria, a matching case living in Syria was randomly chosen. We only matched for age and gender as other variables would vary between the countries such as salaries and the socioeconomic status standards.

The Screen for Post-traumatic Stress Symptoms (SPTSS) tool was used for PTSS assessment which does not require a single traumatic event and screens for PTSS (Aljurany, 2013; Kakaje et al., 2020a). Multidimensional Scale of Perceived Social Support (MSPSS) for social support (R and Kazarian, 2012; Zimet, 2016) and Kessler 10 (K10) for anxiety and depression were also used (Andrews and Slade, 2001; Kessler et al., 2002; Palazón-Bru et al., 2018). These methods were validated in Arabic and previously used in the Syrian community (Kakaje et al., 2020a).

Income was assessed based on the income being adequate for needs rather than how much was earned (Kakaje et al., 2020a). Nearby countries category involved countries that surrounded Syria which were Lebanon, Jordan, Turkey, Iraq, and Iran. Data were processed using IBM SPSS software version 26 for Windows.

Due to the short time of data collecting and the urgency of COVID-19, a larger sample size was not achievable. Around 241 participants from outside Syria received the survey, but only 236 agreed to participate. A larger number of people agreed to participate from Syria. However, only one matching participant was randomly chosen whereas the others were excluded. This study included 472 participants, of which $214(45.3 \%)$ were males with a mean age of $29.1 \pm 7.5$ years.

There were no significant differences in SPTSS, K10, and social support scores between different governorates within Syria $(p>0.05)$. Living in Syria was associated with a higher level of education, owning houses or apartments rather than tenants, being singles, and having less monthly income adequacy compared to living in other countries $(p<0.001)$. Results of the scales are demonstrated in Table 1. When applying cut-off points, Syrians in Syria comprised of $72(31.3 \%)$ with avoidance symptoms, 106 (46.5\%) with arousal symptoms, and $103(45.4 \%)$ with re-experience symptoms, whereas Syrians outside Syria comprised of 96 (41.6\%) with avoidance symptoms, $112(49.6 \%)$ with arousal symptoms, and $111(50.5 \%)$ with re-experience symptoms. Therefore, only avoidance was significantly more frequent in Syria $(p=0.022)$.

Doctors and dentists living in Syria had higher scores than the others compared to living in other countries.

Participants from Syria were more worried from the reduced ability to earn money, being unable to provide food, and their relationship with their deteriorated friends or family ( $p<$ $0.05)$, but they were less worried about getting infected $(p=0.041)$ compared with Syrians outside. However, there were no significant differences in the relationship with the partner and housemates, being distressed from war noise, being themselves or family exposed/infected to COVID-19, being hospitalized, self-quarantined with or without symptoms, losing jobs, and worries of work and study being affected. K10 score was not statistically significantly different according to the type of work.

COVID-19 outbreak was associated with higher PTSS for Syrians outside Syria, which might be from Syria having fewer cases and being more stable socially as they lived with 


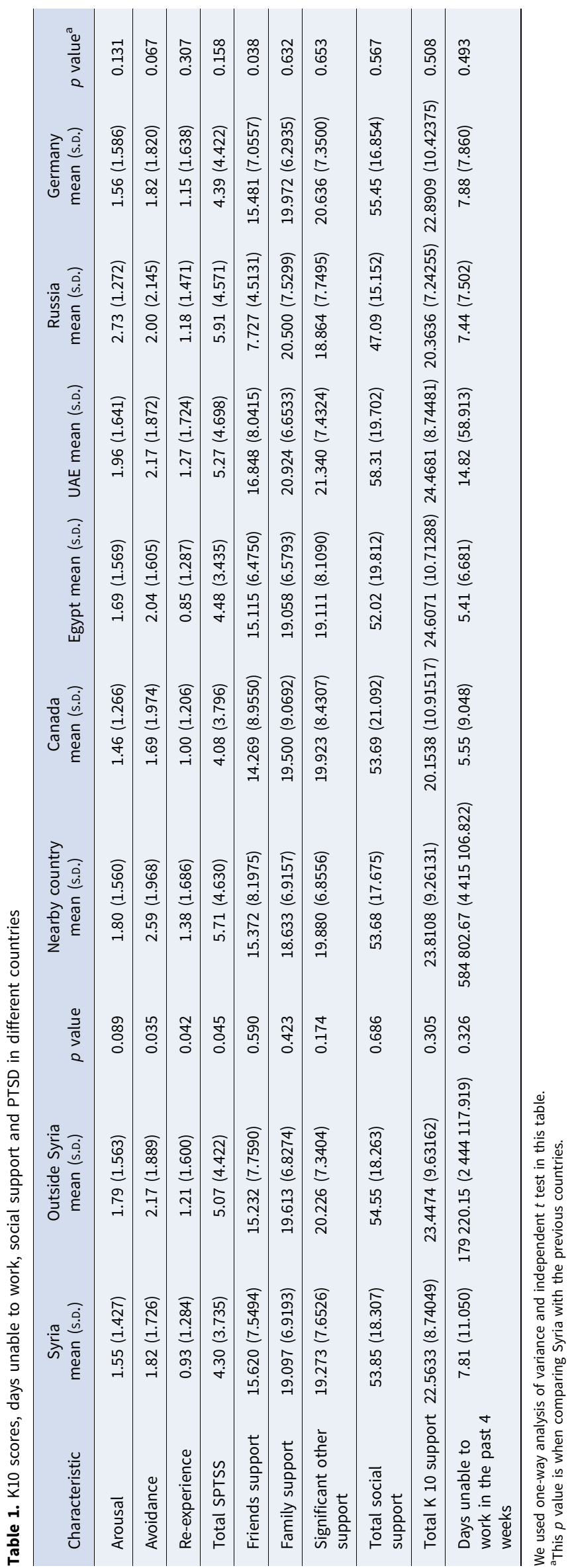

their families in their houses, not in a foreign country. This could also be from the 4-week lockdown providing a break for people, which helped in decreasing their symptoms in Syria.

New methods should be introduced to target Syrian populations as up-to-date and accurate data can be protective against mental distress (Alyousbashi and Almahayni (n.d); Luo et al., 2020). This is particularly prominent in societies such as Syria as the majority of people are daily users of social media and therefore it will provide an easy and fast way to reach the population. The majority of Syrians get their information from social media which leaves a large window of false information. It was difficult to reach the targeted population in our study, so online surveys in social media groups were used which may expose to bias and might have made it more difficult to compare with other samples. However, social media has proven to be a viable item for global mental health which allowed us to reach populations, easier and faster, but we still need to develop the tools to tackle this multi-dynamic crisis. This also justifies the high response rate as these are people who had time and the surveys were easy to fill in, which might have also biased the results as the people who did not have time or Internet access would not have seen the surveys.

In conclusion, as Syria did not have many active cases and the conflict was relatively less severe at the time of this study, Syrians in Syria were particularly concerned about providing food and a reduced ability to earn. This study suggests that Syrians who had to leave and live in a foreign country where COVID-19 outbreak started to rise faced a different mental distress than those who stayed during the war despite both groups having roughly the same social support. This study suggests that mental issues inside Syria are mostly from the deteriorating economy whereas for Syrians outside suffered mostly from COVID-19. In a time of accelerating, ongoing, dynamically overlapping, and rapidly evolving global crises such as COVID-19, more nimble and versatile ways of tracking mental health change longitudinally and comparatively are needed.

Data. The data can be made available upon a reasonable request.

Author contributions. AK: conceptualization; data curation; formal analysis; investigation; methodology; project administration; supervision; resources; validation; original draft; writing, review and editing. AF: review and editing; original draft; investigation; software; resources. AG: conceptualization; project administration; editing; software. RAZ: project administration; software; investigation. All authors have read and approved the manuscript.

Financial support. No funding was received for this study. We did not receive any support in the forms of grants, equipment, or drugs.

Conflict of interest. We have no conflict of interest to declare.

Ethical standards. Online informed consent was taken before proceeding with the survey for participating in the research, and for using and publishing the data. We assured to maintain confidentiality and asked no questions that might reveal the person's identity. No subjects were under the age of 16 . Our study protocol and ethical aspects were reviewed and approved by Damascus University deanship, Damascus, Syria.

Consent for publication. Online consent for using and publishing the data was taken before participating in the research.

\section{References}

Aljurany KAH (2013) Personality characteristics, trauma and symptoms of PTSD: a population study in Iraq. Heriot-Watt University. 
Alyousbashi A, and Almahayni R (Preprint) A cross-section study to assess Health Information Seeking Behavior (HISB) in Syria.

Andrews G, and Slade T (2001) Interpreting scores on the Kessler psychological distress scale (K10). Australian and New Zealand Journal of Public Health 25, 494-497.

Georgiadou E, Zbidat A, Schmitt GM and Erim Y (2018) Prevalence of mental distress among Syrian refugees with residence permission in Germany: a registry-based study. Frontiers in Psychiatry 9, 393.

Kakaje A, Zohbi RA, Aldeen OH, Makki L, Alyousbashi A, and Alhaffar BA (2020a) Mental disorder and PTSD in Syria during wartime: a nationalwide crisis.

Kakaje A, Zohbi RA, Alyousbashi A, Abdelwahed RNK, Aldeen OH, Alhalabi MM, Ghareeb A, and Latifeh Y (2020b) Mental health of school students in Syria after nine years of conflict: a school-based study.
Kessler RC, Andrews G, Colpe LJ, Hiripi E, Mroczek DK, Normand SLT, Walters EE, and Zaslavsky AM (2002) Short screening scales to monitor population prevalences and trends in non-specific psychological distress. Psychological Medicine 32, 959-976.

Luo M, Guo L, Yu M, Jiang W and Wang H (2020) The psychological and mental impact of coronavirus disease 2019 (COVID-19) on medical staff and general public - a systematic review and meta-analysis. Psychiatry Research 291, 113190.

Merhi R and Kazarian SS (2012) Validation of the Arabic translation of the multidimensional scale of social support (Arabic MSPSS) in a Lebanese community sample. Arab Journal of Psychiatry 23, 159-168.

Palazón-Bru A, Sampasa-Kanyinga H, Zamorski MA and Colman I (2018) The psychometric properties of the 10-item Kessler psychological distress scale (K10) in Canadian military personnel. PLoS One 13, e0196562.

Zimet G (2016) Multidimensional Scale of Perceived Social Support (MSPSS) - Scale Items and Scoring Information. 